\title{
Quarkonium measurements in heavy-ion collisions with the STAR experiment
}

\author{
Xinjie Huang ${ }^{1}$ on behalf of the STAR Collaboration \\ ${ }^{1}$ Tsinghua University, Beijing 100084, China
}

\begin{abstract}
In these proceedings, we present the latest measurements of $J / \psi$ and $\Upsilon$ by the STAR experiment. The $J / \psi$ and $\Upsilon$ production measured in $\mathrm{p}+\mathrm{p}$ collisions provide new baselines for similar measurements in $\mathrm{Au}+\mathrm{Au}$ collisions, while the measurements in p+Au collisions can help quantify the cold nuclear matter effects. The $J / \psi v_{2}$ is measured in both $\mathrm{U}+\mathrm{U}$ and $\mathrm{Au}+\mathrm{Au}$ collisions to place constraints on the amount of $J / \psi$ arising from recombination of deconfined charm and anti-charm pairs. Furthermore, the nuclear modification factors for ground and excited $\Upsilon$ states as a function of transverse momentum and centrality are presented, and compared to those measured at the LHC as well as to theoretical calculations.
\end{abstract}

\section{Introduction}

Quarkonium suppression in the medium due to the color screening effect has been proposed as a direct signature of the formation of the Quark Gluon Plasma (QGP) [1]. However, other effects, such as cold nuclear matter (CNM) effects and regeneration of quarkonium states from deconfined heavy quark pairs, give rise to additional complications in the interpretation of the observed suppression. Measurements of the $J / \psi$ elliptic flow $\left(v_{2}\right)$ in different collision systems can help disentangle the different sources contributing to the observed $J / \psi$ population. Compared to charmonia, bottomonia receive less regeneration contribution due to the smaller bottom quark cross-section, thus providing a cleaner probe. Furthermore, different bottomonium states with their different binding energies are expected to dissociate at different temperatures. Measurements of this "sequential melting" can help constrain the medium temperature.

In these proceedings, we present the lastest measurements of $J / \psi$ and $\Upsilon$ productions via both the dimuon and dielectron decay channels. The dimuon channel measurements are based on data samples triggered by the Muon Telescope Detector (MTD) corresponding to an integrated luminosity of 14.2 $\mathrm{nb}^{-1}$ for $\mathrm{Au}+\mathrm{Au}$ collisions at $\sqrt{s_{\mathrm{NN}}}=200 \mathrm{GeV}$ from the RHIC 2014 run, and integrated luminosities of $122 \mathrm{pb}^{-1}$ for $\mathrm{p}+\mathrm{p}$ collisions and $409 \mathrm{nb}^{-1}$ for $\mathrm{p}+\mathrm{Au}$ collisions at $\sqrt{s_{\mathrm{NN}}}=200 \mathrm{GeV}$ from the RHIC 2015 run. The dielectron channel measurements are based on data triggered by the Barrel ElectroMagnetic Calorimeter (BEMC) corresponding to an integrated luminosity of $1.1 \mathrm{nb}^{-1}$ for $\mathrm{Au}+\mathrm{Au}$ collisions at $\sqrt{s_{\mathrm{NN}}}=200 \mathrm{GeV}$ from the RHIC 2011 run, and integrated luminosities of $97 \mathrm{pb}^{-1}$ for $\mathrm{p}+\mathrm{p}$ collisions and $300 \mathrm{nb}^{-1}$ for $\mathrm{p}+\mathrm{Au}$ collisions at $\sqrt{s_{\mathrm{NN}}}=200 \mathrm{GeV}$ from the RHIC 2015 run.

\section{Results}

In all figures presented in this section, statistical uncertainties are shown as vertical bars while systematic uncertainties are shown as open boxes or brackets around the data points. Filled boxes around unity represent the global uncertainties. 
The left panel of Fig. 1 shows the inclusive $J / \psi$ cross-section scaled by the branching ratio $B$ and measured via the dimuon channel in the transverse momentum $\left(p_{\mathrm{T}}\right)$ range of $1<p_{T}<10 \mathrm{GeV} / c$ (red circles) and via the dielectron channel in $0<p_{T}<14 \mathrm{GeV} / c$ (blue squares) in $\mathrm{p}+\mathrm{p}$ collisions at $\sqrt{s_{\mathrm{NN}}}=200 \mathrm{GeV}$. The results are consistent in the overlaping $p_{T}$ range, and can be well discribed by CGC+NRQCD [2] and NLO NRQCD [3] predictions for prompt $J / \psi$ production at low and high $p_{T}$, respectively. An improved color evaporation model (ICEM) for direct $J / \psi$ production [4] describes the data in $p_{T}<3 \mathrm{GeV} / c$, but underestimates the yields at higher $p_{T}$.

The right panel of Fig. 1 shows the $R_{\mathrm{pAu}}$, which quantifies the CNM effects, as a function of $p_{T}$. The $R_{\mathrm{pAu}}$ is less than unity at low $p_{T}$, and consistent with unity within uncertainties at high $p_{T}$. The data is compared with theoretical calculations with the nuclear PDF effect only (color bands) [5-7] and with an additional effect of nuclear absorption (blue dashes) [8]. The calculation with an additional effect of nuclear absorption is favored by the data.
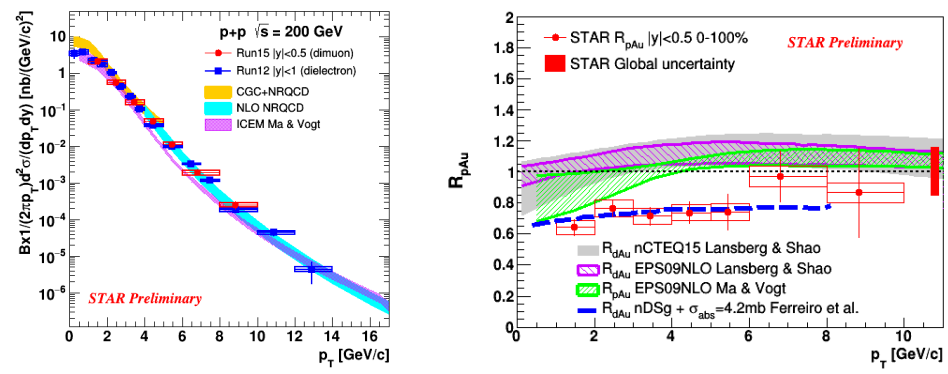

Figure 1. Left: Inclusive $J / \psi$ cross-section in $\mathrm{p}+\mathrm{p}$ collisions at $\sqrt{s_{\mathrm{NN}}}=200 \mathrm{GeV}$ measured via the dimuon (red circles) and dielectron channels (blue squares) as a function of $p_{T}$. Right: Inclusive $J / \psi R_{\mathrm{pAu}}$ as a function of $p_{T}$, compared with theoretical calculations [5-8].

Figure 2 shows the $J / \psi v_{2}$ as a function of $p_{T}$. The new result for $\mathrm{U}+\mathrm{U}$ collisions at $\sqrt{s_{\mathrm{NN}}}=193$ $\mathrm{GeV}$ (red circles) from 2012 data is consistent within error bars with the result for $\mathrm{Au}+\mathrm{Au}$ collision at $\sqrt{s_{\mathrm{NN}}}=200 \mathrm{GeV}$ (black circles) from 2010 and 2011 data [9]. For $p_{T}>2 \mathrm{GeV} / \mathrm{c}, v_{2}$ is consistent with zero within uncertainties, indicating that the contribution from regeneration of fully thermalized charm quarks to $J / \psi$ is likely to be small.

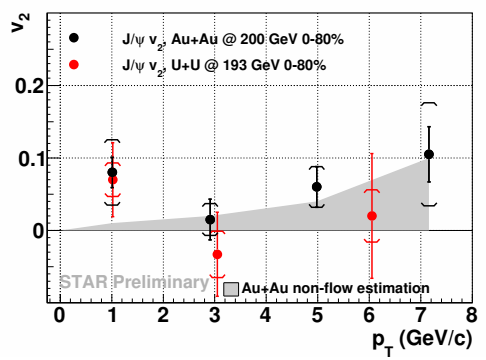

Figure 2. $J / \psi v_{2}$ as a function of $p_{T}$ measured in $\mathrm{U}+\mathrm{U}$ collisions at $\sqrt{s_{\mathrm{NN}}}=193 \mathrm{GeV}$ (red circles) and $\mathrm{Au}+\mathrm{Au}$ collisions at $\sqrt{s_{\mathrm{NN}}}=200 \mathrm{GeV}$ (black circles) [9]. The grey band shows the estimated non-flow contribution.

With BEMC-triggered data from 2015, the $\Upsilon(1 \mathrm{~S}+2 \mathrm{~S}+3 \mathrm{~S})$ production cross-section in $\mathrm{p}+\mathrm{p}$ collisions at $\sqrt{s}=200 \mathrm{GeV}$ within $|y|<0.5$ is measured to be $B \cdot \mathrm{d} \sigma / \mathrm{dy}=81 \pm 5$ (stat.) \pm 8 (syst.) pb, where $B$ is the branching ratio. The new result of better precision is consistent with the published STAR result [10], and follows the trend of world-wide experimental data as well as NLO CEM predictions, as can be seen in the left panel of Fig. 3 . 
The right panel of Fig. 3 shows the $\Upsilon(1 \mathrm{~S}+2 \mathrm{~S}+3 \mathrm{~S}) R_{\mathrm{pAu}}$ as a function of rapidity. The new results measured with BEMC-triggered data from 2015 (red stars) are consistent with the published $R_{\mathrm{dAu}}$ (red circles) but with smaller relative uncertainties. The $R_{\mathrm{pAu}}$ within $|y|<0.5$ is $0.82 \pm 0.10$ (stat. ${ }_{+0.08}^{-0.07}$ (syst.) \pm 0.10 (norm.), hinting at sizable CNM effects.
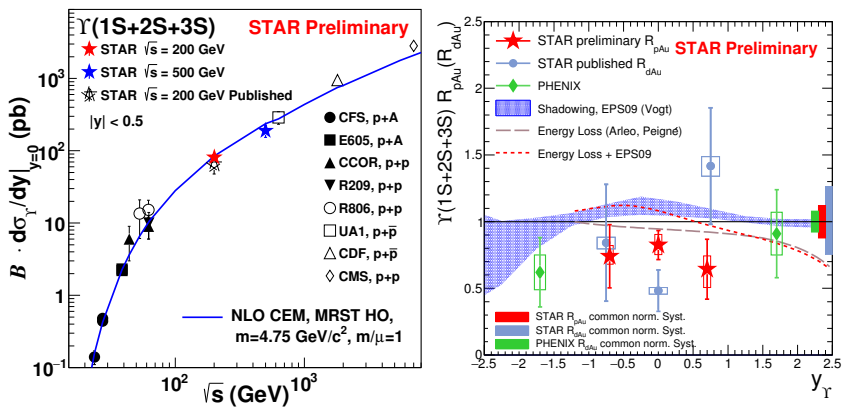

Figure 3. Left: $\Upsilon(1 S+2 S+3 S)$ production cross-section measured in $\mathrm{p}+\mathrm{p}$ collision at $\sqrt{s}=200 \mathrm{GeV}$ (red star) and $\sqrt{s}=500 \mathrm{GeV}$ (blue star) compared with world-wide data and NLO CEM predictions [11]. Right: the $\Upsilon(1 \mathrm{~S}+2 \mathrm{~S}+3 \mathrm{~S}) R_{\mathrm{pAu}}$ (red stars) as a function of rapidity compared to STAR and PHENIX published $R_{\mathrm{dAu}}$ results.

The $\Upsilon(1 \mathrm{~S}+2 \mathrm{~S}+3 \mathrm{~S}) R_{\mathrm{AA}}$ in $\mathrm{Au}+\mathrm{Au}$ collisions at $\sqrt{s_{\mathrm{NN}}}=200 \mathrm{GeV}$ has been measured via the dimuon channel with MTD-triggered data from 2014 and the dielectron channel with BEMC-triggered data from 2011. Since the nuclear modification factors in both channels are consistent with each other within uncertainlies, they are combined to improve the precision.

The centrality dependence of the combined $R_{\mathrm{AA}}$ for $\Upsilon(1 \mathrm{~S})$ and $\Upsilon(2 \mathrm{~S}+3 \mathrm{~S})$ is shown in the left and middle panels of Fig. 4, compared with the lastest CMS results [12]. $\Upsilon(2 S+3 S)$ are more suppressed in central collisions than $\Upsilon(1 \mathrm{~S})$, which is consistent with the sequential melting. Comparing RHIC and LHC results, the levels of suppression for $\Upsilon(1 \mathrm{~S})$ are consistent, while $\Upsilon(2 \mathrm{~S}+3 \mathrm{~S})$ seem to be less suppressed at the RHIC than that at the LHC.
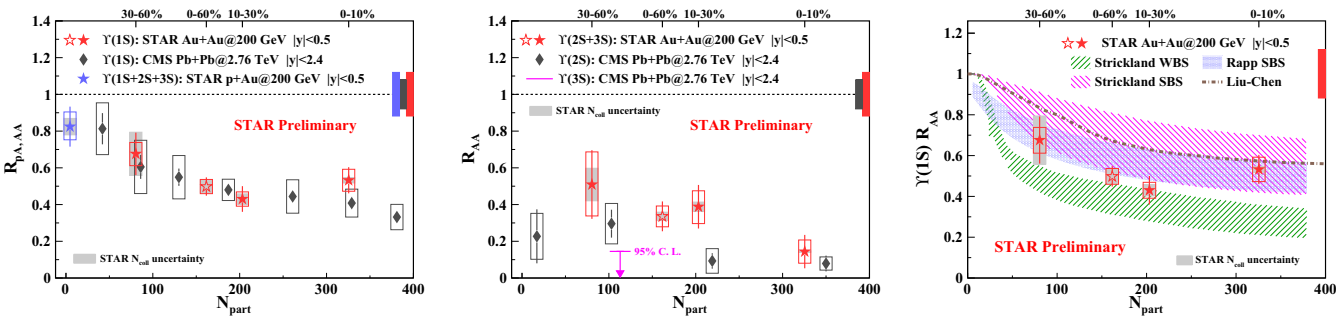

Figure 4. The $R_{\mathrm{AA}}$ of $\Upsilon(1 \mathrm{~S})$ (left panel) and $\Upsilon(2 \mathrm{~S}+3 \mathrm{~S})$ (middel panel) as a function of $N_{\text {part }}$ in Au+Au collisions at $\sqrt{s_{\mathrm{NN}}}=200 \mathrm{GeV}$ for $|y|<0.5$ (red stars), together with the STAR $R_{\mathrm{pAu}}$ for $\Upsilon(1 \mathrm{~S}+2 \mathrm{~S}+3 \mathrm{~S})$ within $|y|<0.5$ (blue star) and the lastest CMS results in $\mathrm{Pb}+\mathrm{Pb}$ collisions at $\sqrt{s_{\mathrm{NN}}}=2.76 \mathrm{TeV}$ within $|y|<2.4$ (black diamonds). Right: The combined $R_{\mathrm{AA}}$ of $\Upsilon(1 \mathrm{~S})$ within $|y|<0.5$ compared to different model calculations [13-15]

The right panel of Fig. 4 shows the centrality dependence of the combined $\Upsilon(1 \mathrm{~S}) R_{\mathrm{AA}}$ together with three SBS (strongly bound scenario) model calculations which use internal-energy-based heavy quark potential and a WBS (weakly bound scenario) model calculation which uses free-energy-based heavy quark potential. The Strickland-Bazow model [13], which takes into account the feed down contributions from excited bottomonium states, studies both scenarios but includes neither CNM nor regeneration effect. The Liu-Chen model [14] considers only the dissociation of the excited states. The Emerick-Zhao-Rapp SBS model [15] accounts for CNM effects and the regeneration contribution. The SBS models are favored by data, especially in central collisions. 
The $p_{T}$ dependence of the $R_{\mathrm{AA}}$ for $\Upsilon(1 \mathrm{~S})$ and $\Upsilon(2 \mathrm{~S}+3 \mathrm{~S})$ measured via the dimuon channel is shown in the left and right panels of Fig. 5, compared with the lastest CMS results [12]. Again for $\Upsilon(1 \mathrm{~S})$ the suppression is consistent at the RHIC and the LHC, while for $\Upsilon(2 \mathrm{~S}+3 \mathrm{~S})$ the suppression seems to be stronger at high- $p_{T}$ at the LHC.
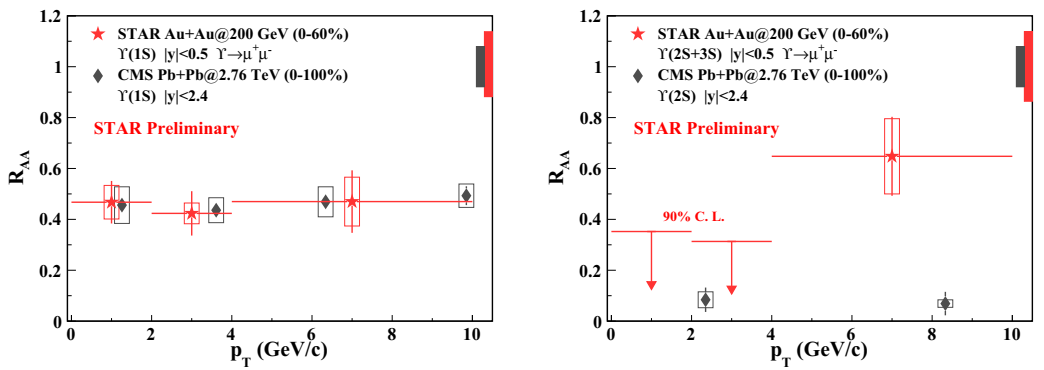

Figure 5. $R_{\mathrm{AA}}$ of $\Upsilon(1 \mathrm{~S})$ (left panel) and $\Upsilon(2 \mathrm{~S}+3 \mathrm{~S})$ (right panel) at mid-rapidity as a function of $p_{\mathrm{T}}$ in $\mathrm{Au}+\mathrm{Au}$ collisions at $\sqrt{s_{\mathrm{NN}}}=200 \mathrm{GeV}$ (red stars) compared with CMS results for $\mathrm{Pb}+\mathrm{Pb}$ collisions at $\sqrt{s_{\mathrm{NN}}}=2.76 \mathrm{TeV}$ (black diamonds).

\section{Summary and Outlook}

In summary, we present the latest measurements of $J / \psi$ and $\Upsilon$ productions in $p+p, p+A u$ and $\mathrm{Au}+\mathrm{Au}$ collisions at $\sqrt{s_{\mathrm{NN}}}=200 \mathrm{GeV}$ and in $\mathrm{U}+\mathrm{U}$ collisions at $\sqrt{s_{\mathrm{NN}}}=193 \mathrm{GeV}$ from the STAR experiment. In $\mathrm{p}+\mathrm{p}$ collisions, the inclusive $J / \psi$ production cross-section can be described by the CGC+NRQCD at low $p_{T}$ and NLO NRQCD at high $p_{T}$. The new $\Upsilon(1 \mathrm{~S}+2 \mathrm{~S}+3 \mathrm{~S})$ production crosssection result with better precision compared to previously published results follows the trend of world-wide experimental data as well as NLO CEM predictions. In p+Au collisions, the $J / \psi R_{\mathrm{pAu}}$ is below unity at low $p_{T}$ and consistent with unity at high $p_{T}$. The $R_{\mathrm{pAu}}$ for $\Upsilon$ within $|y|<0.5$ is measured to be $0.82 \pm 0.10$ (stat. ${ }_{+0.08}^{-0.07}$ (syst.) \pm 0.10 (norm.).

In $\mathrm{U}+\mathrm{U}$ and $\mathrm{Au}+\mathrm{Au}$ collisions, the $J / \psi v_{2}$ results are consistent with each other within uncertainties, and consistent with zero for $p_{T}>2 \mathrm{GeV} / \mathrm{c}$, indicating that the regeneration of fully thermalized charm quarks to $J / \psi$ is unlikely to be the dominant source contributing in this kinematic region. For $\Upsilon$ in Au+Au collisions, the excited $\Upsilon$ states are more suppressed than the ground state in central collisions, which is consistent with the sequential melting. There is also a hint of less suppression at the RHIC than at the LHC for excited $\Upsilon$ states. The statistical precision of quarkonium measurements in $\mathrm{Au}+\mathrm{Au}$ collisions can be further improved by including the similar amount of additional data on tape.

\section{References}

[1] T. Matsui, H. Satz, Phys. Lett. B178 (1986) 416-422.

[2] Ma and Venugopalan,PRL 113 (2014) 192301.

[3] Shao, JHEP 05 (2015) 103.

[4] Ma and Vogt, PRD 94 (2016) 114029.

[5] Ma and Vogt, Private Communication (2017).

[6] Lansberg and Shao, Eur.Phys.J. C77 (2017) no.1, 1.

[7] Shao, Comput. Phys. Commun. 184 (2013) 2562-2570., 198 (2016) 238-259.

[8] Ferreiroet al., Syst. 53 (2012) 27-36.

[9] The STAR Collaboration, PRL 111 (2013) 052301.

[10] The STAR collaboration, Phys. Lett. B 735, 127 (2014).

[11] Frawley, Ullrich, Vogt, Phs. Rev. 462, 125 (2008).

[12] The CMS collaboration, Phys. Lett. B 770, 357 (2017).

[13] Strickland and Bazow, Nucl. Phys. A 879, 25 (2012).

[14] Liu, Chen, Xu, Zhuang, Phys. Lett. B 697, 32 (2011).

[15] Emerick, Zhao, Rapp, Eur. Phys. J. A 48, 72 (2012). 